\title{
Fuel and Run: Metabolism in Neutrophil Migration
}

\author{
Bojing Shao ${ }^{2 *}$, Huiping Shi ${ }^{1}$ and Xindi Shan ${ }^{2}$ \\ ${ }^{1}$ Department of Biochemistry and Molecular Biology, University of Oklahoma Health Sciences Center, USA \\ ${ }^{2}$ Cardiovascular Biology Program, Oklahoma Medical Research Foundation, USA
}

*Corresponding author: Bojing Shao, Cardiovascular Biology Program, Oklahoma Medical Research Foundation, 825 NE. 13th Street, Oklahoma City, OK, 73104.

To Cite This Article: Bojing Shao, Fuel and Run: Metabolism in Neutrophil Migration. Am J Biomed Sci \& Res. 2019 - 6(4). AJBSR.MS.ID.001042. DOI: 10.34297 /AJBSR.2019.06.001042.

Received: November 21, 2019; Published: November 26, 2019

\begin{abstract}
Neutrophils are an essential component of the host innate immune system. Migration of neutrophils into inflicted sites composes the primary defense line against pathogens. Recent studies indicate that neutrophils utilize multiple metabolic pathways, instead of only glycolysis. Importantly, neutrophils may preferentially adopt a certain metabolic pathway to adapt to the altered cellular environment or upon various stimuli. In addition, recent studies have shown mitochondrial and TCA cycle are indispensable for neutrophil migration, and here we briefly discuss the regulation of neutrophil migration through cellular metabolism.
\end{abstract}

\section{Introduction}

Neutrophils, the most abundant circulating leukocytes in human, are the first line of host innate cellular defense against invading microorganisms [1]. Neutrophil migration into the inflicted site is critical for pathogen clearance and tissue repair, as aberrant neutrophil migration into tissues causes recurrent infections [2,3] and tissue damage seen in pathological conditions like ischemiareperfusion injury and arthritis [4]. The recruitment of neutrophils into inflicted tissue is a multiple-step cascade, including neutrophil tethering, rolling, adhesion, crawling, and extravasation. In inflicted tissue neutrophils execute microorganisms through phagocytosis, oxidative burst, cytokines secretion, and neutrophil extracellular traps (NETs). Regulation of neutrophil migration has been excellently reviewed $[1,5,6]$ and here we briefly summarize recent advancements on cellular metabolic insights in neutrophil migration.

\section{Metabolic pathways in neutrophils}

Neutrophil metabolism has not received much attention for long time as neutrophils are terminally differentiated short-lived cells. Previously, glycolysis was considered as the only metabolic pathway in neutrophils [7]. During glycolysis, low levels of Adenosine 5'-triphosphate (ATP) and 1,4-Dihydronicotinamide adenine dinucleotide (NADH) are generated when glucose is converted to pyruvate in the cytosol of neutrophils. Then pyruvate is converted into acetyl coenzyme A (Acetyl CoA) or lactate when in the presence or absence of oxygen, respectively. Acetyl CoA enters tricarboxylic acid (TCA) cycle in the mitochondria to generate ATP efficiently through electron transport chain. The glycolytic intermediate glucose-6-phosphate in the cytosol can be used to generate ribose-5-phosphate and NADPH, which is an essential substrate for the generation of reactive oxygen species (ROS) in activated neutrophils. Recent studies indicate that neutrophils utilize multiple metabolic pathways to fulfill the requirement of energy, biosynthesis, and function. Besides glycolysis, neutrophils also use metabolic pathways like TCA cycle, oxidative phosphorylation, pentose phosphate pathway (PPP), and fatty acid oxidation (FAO) [7-10]. Thus, carbohydrates, proteins, lipids, and amino acids can all be metabolic fuels for neutrophils. Multiple metabolic routes ensure neutrophils to obtain energy and function properly under different pathological conditions. For example, the plasma triglyceride level is elevated during bacterial and viral infection, while total cholesterol, LDL cholesterol, and HDL cholesterol levels are reduced [11-14]. Meanwhile, neutrophils are capable of altering their metabolism to adapt to the changed environment. For instance, resting and activated neutrophils express glucose transporters GLUT1, GLUT3 and GLUT4 differently [15]. Under glucose-limiting conditions, neutrophils utilize fatty acids and glutamine to generate acetyl CoA through mitochondrial 
fatty acid $\beta$ oxidation and glutamine oxidation, respectively $[16,17]$. Thus, metabolic plasticity helps neutrophils survive and function under diverse stresses.

\section{Metabolic regulation of neutrophil transmigration}

Neutrophil migration is important for the removal of invaded pathogens and healing of injured tissues during immune responses. During migration, neutrophils consume huge amount of energy, particularly for the cytoskeleton reorganization events like neutrophil adhesion, polarization, and crawling $[1,2,5,6]$. Coupling with the actin cytoskeleton is critical for integrindependent mechanotransduction, which is required for neutrophil firm adhesion [18,19]. Neutrophils are polarized when cells are crawling on the surface of blood endothelial cells by forming lamellipodia in the leading edge and uropod at the tail, which are enriched with actin and myosin, respectively. It is believed that polarization is essential for directional migration of neutrophils [20]. The cytoskeleton reorganization is regulated by small GTPases $[21,22]$ which commonly requires energy to function properly [23]. It has been reported that mitochondrial and TCA enzymes regulate neutrophil migration [24,25]. Treating neutrophils with an uncoupler of mitochondrial oxidative phosphorylation FCCP [26] or mutating iso-citrate dehydrogenase (IDH), an enzyme in the TCA cycle metabolism [27] reduces ATP production and leads to impairment of neutrophil migration. Studies have revealed that ATP released at the leading edge of crawling neutrophils amplifies chemokine signaling and controls gradient sensing and migration through P2Y2 and A3 receptors in an autocrine feedback manner [28]. Further studies have shown that binding of ATP to P2Y2 also promotes mTOR signaling to enhance mitochondrial activity at the leading edge of neutrophils [24]. In summary, mitochondria and the TCA cycle regulate neutrophil migration through producing ATP that is required to reorganize the cytoskeleton during neutrophil migration.

\section{Future Prospects}

There is growing amount of evidence indicating that neutrophils utilize multiple metabolic pathways, and cellular metabolism is critical for neutrophil migration. However, many questions still remain to be addressed in this cutting-edge field. For example, the mechanism underlying metabolic switch in neutrophils under different pathological conditions is not well studied. Migration of neutrophils into inflamed tissue and healthy tissue (homeostasis) shares a lot of similarities. Thus, whether metabolism plays a similar role in neutrophil recruitment into inflicted site and tissue homeostasis is not known. Recent studies have identified neutrophils as a heterogenous population with varied cellular plasticities $[29,30]$. The metabolic profiles in different neutrophil subsets have not been characterized. Whether and how a circadian rhythm affects neutrophil metabolism is not clear. Finally, better understanding of metabolism in neutrophil migration could provide insights in the development of novel approaches to cure inflammatory diseases.

\section{Funding}

My research project was sponsored by American Heart Association (18CDA34120009).

\section{References}

1. Ley K, Laudanna C, Cybulsky MI, Nourshargh S (2007) Getting to the site of inflammation: the leukocyte adhesion cascade updated. Nat Rev Immunol 7(9): 678-689.

2. Nathan C (2006) Neutrophils and immunity: challenges and opportunities. Nat Rev Immunol 6(3): 173-182.

3. Amulic B, Cazalet C, Hayes GL, Metzler KD, Zychlinsky A (2012) Neutrophil function: from mechanisms to disease. Annu Rev Immunol 30: 459-489.

4. Kolaczkowska E, Kubes P (2013) Neutrophil recruitment and function in health and inflammation. Nat Rev Immunol 13(3): 159-175.

5. McEver RP (2015) Selectins: initiators of leucocyte adhesion and signalling at the vascular wall. Cardiovasc Res 107(3): 331-339.

6. Lagarrigue F, Kim C, Ginsberg MH (2016) The Rap1-RIAM-talin axis of integrin activation and blood cell function. Blood 128(4): 479-487.

7. Borregaard N, Herlin T (1982) Energy metabolism of human neutrophils during phagocytosis. J Clin Invest 70(3): 550-557.

8. Riffelmacher T, Clarke A, Richter FC, Stranks A, Pandey S, et al. (2017) Autophagy-Dependent Generation of Free Fatty Acids Is Critical for Normal Neutrophil Differentiation. Immunity 47(3): 466-480 e5.

9. Azevedo EP, Rochael NC, Guimaraes-Costa AB, de Souza-Vieira TS, Ganilho J, et al. (2015) A Metabolic Shift toward Pentose Phosphate Pathway Is Necessary for Amyloid Fibril- and Phorbol 12-Myristate 13-Acetate-induced Neutrophil Extracellular Trap (NET) Formation. J Biol Chem 290(36): 22174-22183.

10. Richer BC, Salei N, Laskay T, Seeger K (2018) Changes in Neutrophil Metabolism upon Activation and Aging. Inflammation 41(2): 710-721.

11. Khovidhunkit W, Kim MS, Memon RA, Shigenaga JK, Moser AH, et al. (2004) Effects of infection and inflammation on lipid and lipoprotein metabolism: mechanisms and consequences to the host. J Lipid Res 45(7): 1169-1196.

12. Cappi SB, Noritomi DT, Velasco IT, Curi R, Loureiro TC, et al. (2012) Dyslipidemia: a prospective controlled randomized trial of intensive glycemic control in sepsis. Intensive Care Med 38(4): 634-641.

13. van Leeuwen HJ, Heezius EC, Dallinga GM, van Strijp JA, Verhoef J, et al. (2003) Lipoprotein metabolism in patients with severe sepsis. Crit Care Med 31(5): 1359-1366.

14. Gallin JI, Kaye D, O Leary WM (1969) Serum lipids in infection. N Engl J Med 281(20):1081-1086.

15. Maratou E, Dimitriadis G, Kollias A, Boutati E, Lambadiari V, et al. (2007) Glucose transporter expression on the plasma membrane of resting and activated white blood cells. Eur J Clin Invest 37(4): 282-290.

16. Newsholme P, Curi R, Pithon Curi TC, Murphy CJ, Garcia C, et al. (1999) Glutamine metabolism by lymphocytes, macrophages, and neutrophils: its importance in health and disease. J Nutr Biochem 10(6): 316-324.

17. Curi TC, De Melo MP, De Azevedo RB, Zorn TM, Curi R (1997) Glutamine utilization by rat neutrophils: presence of phosphate-dependent glutaminase. Am J Physiol 273(4): C1124-1129.

18. Sun Z, Guo SS, Fassler R (2016) Integrin-mediated mechanotransduction. J Cell Biol 215(4): 445-456. 
19. Chakraborty S, Banerjee S, Raina M, Haldar S (2019) Force-Directed "Mechanointeractome" of Talin-Integrin. Biochemistry.

20. Salvermoser M, Begandt D, Alon R, Walzog B (2018) Nuclear Deformation During Neutrophil Migration at Sites of Inflammation. Front Immunol 9 : 2680.

21. Wong K, Pertz O, Hahn K, Bourne H (2006) Neutrophil polarization: spatiotemporal dynamics of RhoA activity support a self-organizing mechanism. Proc Natl Acad Sci U S A 103(10): 3639-3644.

22. Kumar S, Xu J, Kumar RS, Lakshmikanthan S, Kapur R, et al. (2014) The small GTPase Rap1b negatively regulates neutrophil chemotaxis and transcellular diapedesis by inhibiting Akt activation. J Exp Med 211(9): 1741-1758.

23. Sprang SR (2016) Invited review: Activation of G proteins by GTP and the mechanism of Galpha-catalyzed GTP hydrolysis. Biopolymers 105(8): 449-462

24. Bao Y, Ledderose C, Graf AF, Brix B, Birsak T, et al. (2015) mTOR and differential activation of mitochondria orchestrate neutrophil chemotaxis. J Cell Biol 210(7): 1153-1164.

25. Zhou W, Cao L, Jeffries J, Zhu X, Staiger CJ, et al. (2018) Neutrophilspecific knockout demonstrates a role for mitochondria in regulating neutrophil motility in zebrafish. Dis Model Mech 11(3).
26. Fossati G, Moulding DA, Spiller DG, Moots RJ, White MR, et al. (2003) The mitochondrial network of human neutrophils: role in chemotaxis, phagocytosis, respiratory burst activation, and commitment to apoptosis. J Immunol 170(4): 1964-1972.

27. Amankulor NM, Kim Y, Arora S, Kargl J, Szulzewsky F, et al. (2017) Mutant IDH1 regulates the tumor-associated immune system in gliomas. Genes Dev 31(8): 774-786.

28. Chen Y, Corriden R, Inoue Y, Yip L, Hashiguchi N, et al. (2006) ATP release guides neutrophil chemotaxis via P2Y2 and A3 receptors. Science 314(5806):1792-1795

29. Silvestre-Roig C, Hidalgo A, Soehnlein O (2016) Neutrophil heterogeneity: implications for homeostasis and pathogenesis. Blood 127(18): 21732181.

30. Takashima A, Yao Y (2015) Neutrophil plasticity: acquisition of phenotype and functionality of antigen-presenting cell. J Leukoc Biol 98(4): 489-496. 